\title{
Diatomoflórula Perifítica do rio Descoberto - DF e GO, Brasil, Naviculales (Bacillariophyceae): Diploneidineae e Sellaphorineae
}

\author{
Simone Mogami Delgado ${ }^{1,2}$ e Maria das Graças Machado de Souza ${ }^{1}$
}

Recebido em 2/03/2005. Aceito em 5/02/2007

\begin{abstract}
RESUMO - (Diatomoflórula Perifítica do rio Descoberto - DF e GO, Brasil, Naviculales (Bacillariophyceae)): Diploneidineae e Sellaphorineae). O presente estudo enfocou as diatomáceas perifíticas pertencentes às subordens Diploneidineae e Sellaphorineae de Naviculales (Bacillariophyceae) do rio Descoberto, Distrito Federal, Goiás. As coletas foram realizadas em junho e julho/2003 (período de seca) e janeiro e fevereiro/2004 (período de chuva), em cinco estações de amostragem. Foram identificados 16 táxons infragenéricos, uma espécie pertencente à ordem Diploneidineae e as demais à ordem Sellaphorineae. Foram registradas 14 citações pioneiras para o estado de Goiás e 15 citações pioneiras para o Distrito Federal.
\end{abstract}

Palavras-chave: diatomáceas, rio Descoberto, taxonomia, Bacillariophyta, Naviculales

\begin{abstract}
Periphytic diatom flora of the Descoberto River - Distrito Federal and Goiás State, Brazil, Naviculales (Bacillariophyceae): Diploneidineae and Sellaphorineae). The taxa of the suborders Diploneidineae and Sellaphorineae, Naviculales (Bacillariophyceae) are presented as part of a survey of diatoms in the Distrito Federal (DF), Brazil. Samples were collected at five different points along the Descoberto River, one of the main lotic ecosystems in the Distrito Federal during June-July/2003 (dry season) and January-February (rainy season). Sixteen species from both suborders were identified; 14 species were cited for the first time for Goiás state and 15 for the Distrito Federal.
\end{abstract}

Key words: diatoms, Descoberto River, taxonomy, Bacillaryophyta, Naviculales

\section{Introdução}

As diatomáceas constituem um grupo de importância ecológica e econômica (Van den Hoek et al. 1995; Stoermer et al. 1999); contudo, por apresentarem morfologia complexa, nomenclatura própria, alto índice de polimorfismo e o acesso à literatura especializada ser difícil, os estudos taxonômicos realizados no Brasil se concentram, principalmente, nas regiões sul e sudeste (Brassac \& Ludwig 2003). No estado de Goiás e Distrito Federal há poucos trabalhos e isto de deve a escassez de especialistas e pesquisas na área.

Embora o Estado de Goiás não registre nenhuma pesquisa na qual se discutam as questões taxonômicas das diatomáceas, podem-se citar vários estudos florísticos em que foram identificados táxons de Bacillariophyceae.

Macedo-Saidah et al. (1987) fizeram um estudo qualitativo e quantitativo do plâncton do rio Meia-Ponte
(GO) e observaram 135 táxons, entre os quais 76, em nível específico e infra-específico, pertenciam às diatomáceas. Um inventário da ficoflórula de Goiás elaborado por Campos et al. (1990) apresentou a ocorrência de 20 gêneros de diatomáceas. Na Lagoa Santa em Itajá (GO), Contin \& Oliveira (1993) realizaram um levantamento que registrou a ocorrência de 96 táxons de diatomáceas, identificadas em nível específico e infra-específico, sendo apenas uma não determinada em nível de espécie.

Da mesma forma o Distrito Federal (DF) apresenta poucos estudos taxonômicos da diatomoflórula. A maioria dos estudos apresenta as Bacillariophyta identificadas apenas em nível genérico e, apesar de haver um movimento crescente de registros de táxons específicos e infra-específicos, são estudos de natureza limnológica, sem qualquer informação taxonômica. Assim, para o DF, encontram-se apenas três trabalhos de cunho taxonômico: no trabalho de Souza \& Moreira Filho (1999a) foram tratados taxonomicamente as

\footnotetext{
1 Universidade de Brasília, Instituto de Ciências Biológicas, Departamento de Botânica, Campus Universitário Darcy Ribeiro, Asa Norte, C. Postal 04457, 70910-970 Brasília, DF, Brasil

2 Autor para correspondência: sdmogami@yahoo.com.br
} 
famílias Thalassiosiraceae e Eunotiaceae encontradas na Lagoa Bonita, sendo proposta uma nova combinação para o gênero Eunotia e apresentando uma chave dicotômica sobre os táxons encontrados. No segundo trabalho, foram registrados para a Lagoa Bonita, os gêneros Navicula "sensu lato" e Pinnularia, totalizando 12 táxons (Souza \& Moreira Filho 1999b). Finalmente, o terceiro trabalho tratou da proposição de quatro espécies novas para a ciência: Brachysira staurophora Souza \& Compère, Eunotia sennae Souza \& Compère, Kobayasiella mirabilis Souza \& Compère e Pinnularia sulcata Souza \& Compère (Souza \& Compère 1999).

Com o objetivo de ampliar os estudos taxonômicos da diatomoflórula em sistemas lóticos do DF e Goiás, uma vez que há uma escassez de trabalhos nesses ecossistemas (Brassac \& Ludwig 2005), realizou-se um levantamento das espécies pertencentes às subordens Sellaphorineae e Diploneidineae, ordem Naviculales na bacia do rio Descoberto.

\section{Material e métodos}

O rio Descoberto é usado como fonte de abastecimento de $65 \%$ da população do DF, além de ser utilizado como ponto de lazer da comunidade. Ele nasce à altura da cota de $1.300 \mathrm{~m}$, sob as coordenadas de $15^{\circ} 37^{\prime} \mathrm{S}$ e $48^{\circ} 10^{\prime} \mathrm{W}$, durante seu curso é represado formando a barragem do Descoberto, nas coordenadas de $15^{\circ} 47^{\prime} \mathrm{S}$ e $48^{\circ} 11^{\prime} \mathrm{W}$ (CODEPLAN 1984). Na direção sul, a montante da cidade de Santo Antônio do Descoberto (GO), o rio Descoberto recebe o seu principal afluente, o rio Melchior, que atravessa uma Área de Relevante Interesse Ecológico (ARIE) criada com o intuito de preservar as nascentes dos córregos do Cortado e Taguatinga. No rio Melchior é lançado esgoto doméstico e hospitalar das cidades de Taguatinga, Ceilândia e Samambaia, região de maior densidade demográfica do DF. Além disso, nele localizase a Estação de Tratamento de Esgoto de Samambaia (ETE-CAESB - Samambaia Norte).

A análise da diatomoflórula foi realizada com base em 17 amostras provenientes de cinco estações distribuídas ao longo do rio Descoberto (Tabela 1). Foram realizadas quatro coletas mensais: dois meses de menores índices de pluviosidade (junho e julho/2003) e dois meses de maiores índices pluviométricos (janeiro e fevereiro/2004), caracterizando respectivamente a estação seca e chuvosa, típicas de clima tropical chuvoso de savana (Köppen Aw e Caw) do Distrito
Federal (CODEPLAN 1984). A coleta de perifíton foi realizada raspando-se substrato rochoso, no mínimo, três rochas e substratos vegetais na ausência de rochas no local. Para a raspagem do material aderido aos substratos foram utilizadas escovas de dente. $\mathrm{O}$ substrato, após ser raspado, foi lavado com água destilada e as amostras coletadas foram armazenadas em frascos de vidro com capacidade para $150 \mathrm{~mL}$, e fixadas no campo com formaldeído (4\%). As amostras (Tab. 1) e lâminas permanentes foram depositadas na coleção ficológica do Herbário da Universidade de Brasília (UB). A técnica para oxidação do material foi a de Simonsen (1974), modificada por Moreira Filho \& Valente-Moreira (1981). Para montagem das lâminas foi utilizado Naphrax (IR: 1,74) como meio de inclusão. Os táxons encontrados foram fotografados com uma máquina digital Sony Cyber-Shot, modelo DSC - P10, resolução de 5.0 megapixels, acoplada ao microscópio. O sistema de classificação utilizado para o enquadramento dos táxons analisados foi o de Round et al. (1990).

\section{Resultados e discussão}

\author{
Diploneidineae D.G. Mann \\ Diploneidaceae D.G. Mann \\ Diploneis C.G. Ehrenberg ex Cleve
}

Diploneis subovalis Cleve, Syn. Navic. Dist. Kong., p.96, pr.1, fig.7, 1894.

Fig. 1

Valva elíptica; extremidades arredondadas, área axial retilínea moderadamente larga; área central ovalada no sentido longitudinal; rafe incluída em uma costela; costelas transapicais levemente radiadas; duas fileiras de aréolas entre as costelas, dispostas em quincôncio. Comprimento: 19,4-35,2 $\mu \mathrm{m}$. Largura: 6,4-16,0 $\mu \mathrm{m}$. Estrias: 7-11/10 $\mu \mathrm{m}$.

Distribuição para o Estado de Goiás e DF: citação pioneira para o Estado de Goiás e DF.

Material examinado: BRASIL. Distrito Federal: a jusante da barragem rio Descoberto, 23/VI/2003, M.G.M. Souza (UB 1496); Goiás: a montante da confluência do rio Melchior com o rio Descoberto, 23/VI/2003, M.G.M. Souza (UB 1497); 7/VII/2003 (UB 1508).

Sellaphorineae D.G. Mann

Sellaphoraceae Mereschokowsky

Fallacia Stickle \& D.G. Mann 
Tabela 1. Estações de coleta e amostras no rio Descoberto, DF e GO, Brasil.

\begin{tabular}{|c|c|c|c|c|}
\hline $\begin{array}{l}\mathrm{N}^{\circ} \text { do Tombo } \\
\text { (UB) }\end{array}$ & Estação de coleta $\left(\mathrm{N}^{\circ}\right)$ & Coordenadas & Substrato & $\begin{array}{c}\text { Período } \\
\text { (seca ou chuva) }\end{array}$ \\
\hline 1489 & 01 - Córrego Barrocão, mais próximo a nascente. & $\begin{array}{l}15^{\circ} 37^{\prime} 51^{\prime \prime} \mathrm{S} \\
49^{\circ} 10^{\prime} 37^{\prime \prime} \mathrm{W}\end{array}$ & Epilítico & Seca \\
\hline 1491 & 02 - a montante da barragem do rio Descoberto & $\begin{array}{l}15^{\circ} 42^{\prime} 30^{\prime \prime S} \\
48^{\circ} 14^{\prime} 01^{\prime \prime W}\end{array}$ & Epilítico & Seca \\
\hline 1496 & 03 - a jusante da barragem do rio Descoberto & $\begin{array}{l}15^{\circ} 52^{\prime} 07^{\prime \prime} \mathrm{S} \\
48^{\circ} 16^{\prime} 42^{\prime \prime W}\end{array}$ & Epilítico & Seca \\
\hline 1497 & $\begin{array}{l}04 \text { - a montante da confluência do rio Melchior com } \\
\text { o rio Descoberto (GO) }\end{array}$ & $\begin{array}{l}15^{\circ} 55^{\prime} 52^{\prime \prime} \mathrm{S} \\
48^{\circ} 16^{\prime} 29^{\prime \prime} \mathrm{W}\end{array}$ & Epilítico & Seca \\
\hline 1498 & $\begin{array}{l}05 \text { - a jusante da confluência do rio Melchior com o } \\
\text { rio Descoberto }\end{array}$ & $\begin{array}{l}16^{\circ} 03^{\prime} 49^{\prime \prime} \mathrm{S} \\
48^{\circ} 16^{\prime} 39^{\prime \prime} \mathrm{W}\end{array}$ & Epifítico & Seca \\
\hline 1500 & 01 - Córrego Barrocão, mais próximo a nascente. & $\begin{array}{l}15^{\circ} 37^{\prime} 51 ’ \mathrm{~S} \\
49^{\circ} 10^{\prime} 37^{\prime \prime} \mathrm{W}\end{array}$ & Epilítico & Seca \\
\hline 1502 & 02 - a montante da barragem do rio Descoberto & $\begin{array}{l}15^{\circ} 42^{\prime} 30^{\prime \prime} \mathrm{S} \\
48^{\circ} 14^{\prime} 01^{\prime \prime W}\end{array}$ & Epilítico & Seca \\
\hline 1507 & 03 - a jusante da barragem do rio Descoberto & $\begin{array}{l}15^{\circ} 52^{\prime} 07^{\prime} \mathrm{S} \\
48^{\circ} 16^{\prime} 42^{\prime \prime W}\end{array}$ & Epilítico & Seca \\
\hline 1508 & $\begin{array}{l}04 \text { - a montante da confluência do rio Melchior com o } \\
\text { rio Descoberto }(\mathrm{GO})\end{array}$ & $\begin{array}{l}15^{\circ} 55^{\prime} 52^{\prime \prime} \mathrm{S} \\
48^{\circ} 16^{\prime} 29^{\prime \prime} \mathrm{W}\end{array}$ & Epilítico & Seca \\
\hline 1509 & $\begin{array}{l}05 \text { - a jusante da confluência do rio Melchior com o } \\
\text { rio Descoberto }\end{array}$ & $\begin{array}{l}16^{\circ} 03 ’ 49 ” \mathrm{~S} \\
48^{\circ} 16^{\prime} 39^{\prime \prime} \mathrm{W}\end{array}$ & Epilítico & Seca \\
\hline 1617 & 01 - Córrego Barrocão, mais próxima a nascente. & $\begin{array}{l}15^{\circ} 37^{\prime} 51 ’ \mathrm{~S} \\
49^{\circ} 10^{\prime} 37^{\prime \prime} \mathrm{W}\end{array}$ & Epilítico & Chuva \\
\hline 1619 & 02 - a montante da barragem do rio Descoberto & $\begin{array}{l}15^{\circ} 42^{\prime} 30^{\prime} \mathrm{S} \\
48^{\circ} 14^{\prime} 01^{\prime \prime W}\end{array}$ & Epilítico & Chuva \\
\hline 1624 & 03 - a jusante da barragem do rio Descoberto & $\begin{array}{l}15^{\circ} 52^{\prime} 07^{\prime \prime} \mathrm{S} \\
48^{\circ} 16^{\prime} 42^{\prime \prime W}\end{array}$ & Epilítico & Chuva \\
\hline 1625 & $\begin{array}{l}05 \text { - a jusante da confluência do rio Melchior com o } \\
\text { rio Descoberto }\end{array}$ & $\begin{array}{l}16^{\circ} 03 ’ 49 ” \mathrm{~S} \\
48^{\circ} 16^{\prime} 39^{\prime \prime} \mathrm{W}\end{array}$ & Epilítico & Chuva \\
\hline 1626 & 01 - Córrego Barrocão, mais próxima a nascente. & $\begin{array}{l}15^{\circ} 37^{\prime} 51 ’ \mathrm{~S} \\
49^{\circ} 10^{\prime} 37^{\prime \prime} \mathrm{W}\end{array}$ & Epilítico & Chuva \\
\hline 1628 & 02 - a montante da barragem do rio Descoberto & $\begin{array}{l}15^{\circ} 42^{\prime} 30^{\prime \prime} \mathrm{S} \\
48^{\circ} 14^{\prime} 01^{\prime \prime W}\end{array}$ & Epilítico & Chuva \\
\hline 1633 & 03 - a jusante da barragem do rio Descoberto & $\begin{array}{l}15^{\circ} 52^{\prime} 07^{\prime \prime} \mathrm{S} \\
48^{\circ} 16^{\prime} 42^{\prime \prime} \mathrm{W}\end{array}$ & Epilítico & Chuva \\
\hline
\end{tabular}

Fallacia insociabilis (Krasske) D.G. Mann, The Diatoms. Biol. \& Morphol. of the genera: p. 668, 1990.

Navicula insociabilis Krasske, Hedwigia 72: 114, pr.3, fig. 17, 1932.

Fig. 2

Valva elíptica, extremidade arredondada; área axial estreita e curva; área central pequena, estreita; rafe filiforme, curva, no interior de uma costela axial proeminente; fissuras proximais da rafe expandidas semelhante a um poro, curvadas para o mesmo lado. Estriação paralela no centro, radiada ao longo da valva. Comprimento: 11,7-14,4 $\mu \mathrm{m}$. Largura: 5,6-6,4 $\mu \mathrm{m}$. Estrias: $17-20 / 10 \mu \mathrm{m}$.

O táxon concorda com a descrição de LangeBertalot et al. (1996), Krammer \& Lange-Bertalot
(1986), Germain (1981) e Hustedt (1959), apesar do limite mínimo para o número de estrias esteja abaixo do encontrado neste trabalho. Krammer \& LangeBertalot (1986) comentam que um táxon próximo é Navicula tenera Hustedt, mas este pode ser diferenciado de Fallacia insociabilis (Krasske) D.G. Mann devido a presença de uma fileira de poros distintos e duplos na costela axial.

Distribuição para o Estado de Goiás e DF: citação pioneira para o Estado de Goiás e DF.

Material examinado: BRASIL. Distrito Federal: a jusante da barragem rio Descoberto, 2/II/2004, S.M. Delgado (UB 1633).

Sellaphorineae D.G. Mann

Sellaphoraceae Mereschokowsky

Sellaphora C. Mereschkowsky 
Sellaphora nyassensis (O. Muller) D.G. Mann, Brit. Phycol. J. 24: 2. 1989.

Navicula nyassensis O. Muller, Bot. Jahrb. 45: 83, pr. I, fig. 5. 1910.

Fig. 3-4

Valva lanceolada a elíptica; extremidades rostradas a ligeiramente subcapitadas, área axial linear, estreita; área central larga, retangular; estrias irregularmente encurtadas; rafe filiforme, extremidades proximais da rafe retas, poros distintos; nódulos terminais expandidos lateralmente; estrias radiadas, indistintamente areoladas. Comprimento: 28,8-39,8 $\mu \mathrm{m}$. Largura: 6,4-8,0 $\mu \mathrm{m}$. Estrias: 19-24/10 $\mu \mathrm{m}$.

Distribuição para o Estado de Goiás e DF: citação pioneira para o Estado de Goiás e DF.

Material examinado: BRASIL. Distrito Federal: a montante da barragem do rio Descoberto, 23/VI/2003, M.G.M Souza. (UB 1491); a jusante da barragem rio Descoberto, 23/VI/2003, M.G.M. Souza (UB 1496), 7/VII/2003 (UB 1507); Goiás: a montante da confluência do rio Melchior com o rio Descoberto, 7/VII/2003, M.G.M. Souza (UB 1508).

Sellaphora pupula (Kützing) Mereschkowsky, Ann. Mag. Nat. Hist., Ser. 7(9):187-188, pr. 4, figs.1-5, 1992.

Navicula pupula Kützing, Bacill., p. 93, est. 30, fig. 40, 1844.

Fig. 5

Valva linear, lanceolada a elíptica; extremidades rostradas, arredondadas ou subcapitadas, presença de espessamento silicoso; área axial linear, estreita; área central larga, forma variável, estrias irregularmente encurtadas; rafe filiforme, extremidades proximais da rafe retas, poros distintos; nódulos terminais expandidos lateralmente; estrias radiadas, indistintamente areoladas. Comprimento: 14,6-41,3 $\mu \mathrm{m}$. Largura: 6,2-8,3 $\mu \mathrm{m}$. Estrias: $11-30 / 10 \mu \mathrm{m}$.

Krammer \& Lange-Bertalot (1986) mencionam que Navicula pupula Kützing apresenta um alto grau de polimorfismo em relação a características como o contorno valvar e as extremidades, o que pode ser observado também em Lange-Bertalot \& Metzeltin (1996).

Distribuição para o Estado de Goiás e DF: citação pioneira para o Estado de Goiás e DF.

Material examinado: BRASIL. Distrito Federal: a jusante da barragem rio Descoberto, 23/VI/2003, M.G.M. Souza (UB 1496), 7/VII/2003 (UB 1507); 19/I/2004, S.M. Delgado (UB 1624); a jusante da confluência do rio Melchior com o rio Descoberto, 23/VI/2003, M.G.M. Souza, (UB 1498); 7/VII/2003 (UB 1509); Goiás: a montante da confluência do rio Melchior com o rio Descoberto, 23/VI/2003, M.G.M. Souza (UB 1497).

Sellaphora rectangularis (Gregory) Lange-Bertalot \& Metzeltin, Icon. Diatomol. 2: p. 102, pr.25, figs.10-12, 1996.

Stauroneis rectangularis Gregory, Quart. J. Mikr. Sc. 2, p. 99, pr. 4, fig.17, 1854.

Fig. 6

Valva linear; extremidades largo-arredondadas; área axial linear, estreita; área central irregular, forma variável, estrias irregularmente encurtadas; rafe filiforme, reta; extremidades proximais da rafe retas, poros distintos; estrias radiadas. Comprimento: 33,6-44,2 $\mu \mathrm{m}$. Largura: 8,6-10,7 $\mu \mathrm{m}$. Estrias: 20-21/10 $\mu \mathrm{m}$.

Distribuição para o Estado de Goiás e DF: citação pioneira para Goiás e DF.

Material examinado: BRASIL. Distrito Federal: a jusante da barragem rio Descoberto, 23/VI/2003, M.G.M. Souza (UB 1496); Goiás: a montante da confluência do rio Melchior com o rio Descoberto, 7/VII/2003, M.G.M. Souza (UB 1508).

Sellaphora seminulum (Grunow) D.G. Mann, Brit. Phycol. J. 24: 2, 1989.

Navicula seminulum Grunow, Verh. Zool. - Bot. Ges.

Wien 10:552, pr.4, fig.3, 1860.

Fig. 7

Valva lanceolada, elíptica; extremidades largoarredondadas, agudas; área axial linear, estreita; área central irregular, forma variável; estrias irregularmente encurtadas; rafe filiforme, reta a levemente defletida para o mesmo lado; extremidades proximais da rafe retas, poros distintos; estrias levemente radiadas a paralelas. Comprimento: 8,2-17,3 $\mu \mathrm{m}$. Largura: 3,7-4,8 $\mu \mathrm{m}$. Estrias: 12-20/10 $\mu \mathrm{m}$.

Segundo Krammer \& Lange-Bertalot (1986), N. seminulum e Navicula minima Grunow são táxons próximos que se diferenciam entre si pelo número de estrias, $N$. seminulum com 18 a 22 e $N$. minima com 20 a 25 estrias em $10 \mu \mathrm{m}$. S. seminulum se assemelha com Navicula joubaudii Germain que tem 18 a 20/10 $\mu \mathrm{m}$ estrias (Prygiel \& Coste 1999), mas esta apresenta o contorno elíptico, com ápice subrostrado a arredondado largo, podendo ter uma intumescência na região mediana da valva. 
Distribuição para o Estado de Goiás e DF: citação pioneira para o Estado de Goiás e DF.

Material examinado: BRASIL. Distrito Federal: a jusante da confluência do rio Melchior com o rio Descoberto, 23/VI/2003, M.G.M. Souza (UB 1498); 7/VII/2003 (UB 1509); a jusante da confluência do rio Melchior com o rio Descoberto, 19/I/2004, S.M. Delgado (UB 1625).

Pinnulariaceae D.G. Mann

Caloneis P. Cleve

Caloneis butantana Krasske, Svensk Botanisk

Tidskrift., 42, p. 429. pr.1, fig.36, 1948.

Fig. 8

Valva linear a elíptica, margem paralela; extremidades largo-cuneadas a arredondadas; área axial estreita, regular; área central grande, arredondada, assimétrica; rafe filiforme, reta, poro distinto; fissuras distais da rafe, laterais; estrias paralelas, levemente convergentes na extremidade. Comprimento: 72-101,1 $\mu \mathrm{m}$. Largura: 14,2-15,7 $\mu \mathrm{m}$. Estrias: $13-14 / 10 \mu \mathrm{m}$.

Lange-Bertalot et al. (1996) observam espécimes que denominam de Caloneis butantana Krasske, com medidas de largura e estrias sutilmente abaixo do que o reportado neste trabalho; contudo, as figuras apresentadas por Lange-Bertalot et al. (1996) assemelham-se à população registrada para o rio Descoberto. Metzeltin \& Lange-Bertalot (1998) fazem uma nova combinação para C. butantana, transferindoa para Pinnularia butantanum; entretanto, o material desses mesmos autores não se assemelha com o descrito e ilustrado em Lange-Bertalot et al. (1996) como C. butantana. Portanto, preferiu-se manter o táxon como Caloneis butantana Krasske.

Distribuição para o Estado de Goiás e DF: citação pioneira para Goiás e DF.

Material examinado: BRASIL. Distrito Federal: a jusante da barragem rio Descoberto, 23/VI/2003, M.G.M.Souza (UB 1496); 7/VII/2003 (UB 1507).

Caloneis hyalina Hustedt, Arch. Hydrobiol. Suppl. 15

(2):281, pr. 15, figs.8-10, 1937.

Fig. 9

Valva lanceolada levemente rombóide; extremidades sub-rostradas, arredondadas; área axial estreita; área central larga, retangular, que atinge a margem; rafe filiforme, reta, poros das fissuras proximais distintos; estrias levemente radiadas, delicadas, dificilmente distinguíveis em microscopia de luz. Comprimento: 20,8-24,3 $\mu \mathrm{m}$. Largura: 4,8-5,8 $\mu \mathrm{m}$. Distribuição para o Estado de Goiás e DF: citação pioneira para Goiás e DF.

Material examinado: BRASIL. Distrito Federal: a jusante da confluência do rio Melchior com o rio Descoberto, 7/VII/2003, M.G.M. Souza (UB 1509).

Pinnulariaceae D.G. Mann

Pinnularia C.G. Ehrenberg

Pinnularia amazonica Metzeltin \& Lange-Bertalot, Icon. Diatomol. 5: p. 163, pr.176, fig.: 4-7, 1998.

Fig. 10

Valva linear, margens levemente onduladas, região mediana intumescida; extremidades subcapitadas arredondadas; área axial estreita; área central larga, retangular com fascia; rafe filiforme a levemente lateral; extremidades proximais da rafe voltadas para a mesma direção, poros centrais distintos; extremidade distal da rafe em forma de foice; estrias transapicais radiadas na área central e, em direção às extremidades, paralelas. Comprimento: 102,4 $\mu \mathrm{m}$. Largura: 12,8 $\mu \mathrm{m}$. Estrias: $11 / 10 \mu \mathrm{m}$.

Todas as características encontradas na população observada no rio Descoberto conferem com a descrição de Metzeltin \& Lange-Bertalot (1998), não havendo dúvidas na identificação do táxon como Pinnularia amazonica Metzeltin \& Lange-Bertalot. Metzeltin \& Lange-Bertalot (1998) comentam que Pinnularia amoena Hustedt é semelhante à P. amazonica; contudo, a principal diferença encontra-se nas suas extremidades cuneadas, o que não foi observado no espécime encontrado no rio Descoberto. Pinnularia trigibba Hustedt também é semelhante à espécie descrita por Metzeltin \& LangeBertalot (1998), porém apresenta valva e a área central elíptica a lanceolada, a qual difere de $P$. amazonica. Por fim, Metzeltin \& Lange-Bertalot (1998) comentam que Pinnularia trigonocephala Cleve se assemelha à espécie descrita por eles; no entanto, elas diferem na área central que na $P$. trigonocephala é pequena e arredondada e na espécie $P$. amazonica é larga, retangular e com fascia.

Distribuição para o Estado de Goiás e DF: citação pioneira para Goiás e DF.

Material examinado: BRASIL. Distrito Federal: a jusante da barragem rio Descoberto, 7/VII/2003, M.G.M. Souza (UB 1507).

Pinnularia brauniana (Grunow) Mills, An index of the genera and species of the diatomaceae and their synonyms, 1816-1932.p. 1273, 1934. 
Navicula brauniana Grunow in A. Schmidt et al., Atlas Diatomaceen - KD., pr. 45. fig. 77, 1876.

Fig. 11

Valva linear-elíptico, margens convexas; extremidades capitadas; área axial alargando-se em direção ao centro; área central larga, retangular com fascia; rafe filiforme; extremidades proximais da rafe voltadas para a mesma direção; poros centrais distintos; extremidades distais da rafe em forma de foice; estrias transapicais radiadas na área central e, em direção às extremidades, convergentes. Comprimento: $52,5 \mu \mathrm{m}$. Largura: $8 \mu \mathrm{m}$. Estrias: 10/10 $\mu \mathrm{m}$.

O táxon encontrado concorda com Krammer (1992) e Metzeltin \& Lange-Bertalot (1998). Krammer (1992) coloca Pinnularia braunii (Grunow) Cleve na sinonímia de Pinnularia brauniana (Grunow) Mills e Pinnularia braunii var. amphicephala (Mayer) Hustedt como sinônimo de Pinnularia mayeri Krammer. Um táxon próximo é Pinnularia subcapitata Gregory, porém difere-se de P. brauniana (morfotipo 1) por ter a área axial mais estreita e possuir largura menor (Krammer 1992). Outro táxon semelhante é Pinnularia mesolepta (Ehrenberg) W. Smith, contudo este apresenta valva linear. Por fim, Pinnularia psiciculus var. angusta Metzeltin \& Krammer difere-se de P. brauniana devido a margem reta, com a extremidade acentuadamente capitada e área axial estreita.

Distribuição para o Estado de Goiás e DF: citação pioneira para o DF e Goiás.

Material examinado: BRASIL. Distrito Federal: a jusante da confluência do rio Melchior com o rio Descoberto, 7/VII/2003, M.G.M. Souza (UB 1509).

Pinnularia crucifera Cleve-Euler, Soc. Scient. Fennica, Comment. Biol. 4, 14: p. 48, pr. 3: 68 a, b, 2000.

Fig. 12

Valva linear; margens paralelas; extremidades arredondadas; área axial estreitas; área central larga, lanceolada com fascia estreita; rafe lateral, extremidades proximais da rafe voltadas para a mesma direção; poros centrais distintos, extremidades distais da rafe em forma de foice; estrias transapicais centrais, paralelas; em direção às extremidades, convergentes. Comprimento: 92,8 $\mu \mathrm{m}$. Largura: 13,6 $\mu \mathrm{m}$. Estrias: $9 / 10 \mu \mathrm{m}$.

Distribuição para o Estado de Goiás e DF: citação pioneira para Goiás e DF.

Material examinado: BRASIL. Goiás: a montante da confluência do rio Melchior com o rio Descoberto, 7/VII/2003, M.G.M. Souza (UB 1508).

Pinnularia divergens var. media Krammer, Diatoms of Europe. 1: p. 61. pr. 34, fig. 1-5, 2000.

Fig. 13

Valva levemente linear-lanceolada, margens paralelas a ligeiramente côncavas; extremidades rostradas, subcapitadas a capitadas produzidas; área axial estreita; área central rombóide, presença de fascia e de espessamentos hialinos em forma de meia lua; estrias encurtando-se na região central em ambos os lados da valva; rafe levemente lateral; extremidades proximais da rafe voltadas para a mesma direção; extremidades distais da rafe em forma de baioneta; estrias transapicais alveoladas radiais na região mediana $\mathrm{e}$, em direção às extremidades, levemente paralelas a convergentes. Comprimento: $42,2-67,2 \mu \mathrm{m}$. Largura: 7,7-11,2 $\mu \mathrm{m}$. Estrias: 9-13/10 $\mu \mathrm{m}$.

Distribuição para o Estado de Goiás e DF: citação pioneira para o Estado de Goiás e DF.

Material examinado: BRASIL. Distrito Federal: a jusante da barragem rio Descoberto, 23/VI/2003, M.G.M. Souza (UB 1496); 7/VII/2003 (UB 1507); a jusante da confluência do rio Melchior com o rio Descoberto, 7/VII/2003, M.G.M. Souza (UB 1509); 19/I/2004, S.M. Delgado (UB 1624); 19/I/2004 (UB 1625); a montante da barragem do rio Descoberto, 16/II/2004, S.M. Delgado (UB 1628); Goiás: a montante da confluência do rio Melchior com o rio Descoberto, 23/VI/2003, M.G.M. Souza (UB 1497); 7/VII/2003 (UB 1508).

Pinnularia gibba Ehrenberg, Abh. K. Akad. Wiss. Berlin, 1: 384, pr.211, fig.24; pr.311, fig.4, 1841.

Fig. 14

Valva linear a lanceolada, margens levemente onduladas; extremidades rostradas, sub-rostradas, arredondadas; área axial alargando-se em direção a área central; área central larga, rombóide com fascia; estrias encurtando-se em direção à área central; rafe filiforme a levemente lateral; extremidades proximais da rafe voltadas para a mesma direção; extremidades distais da rafe em forma de ponto-de-interrogação; estrias transapicais radiadas na área central e, em direção às extremidades, paralelas à levemente convergentes. Comprimento: 44,6-64 $\mu \mathrm{m}$. Largura: 10,6-11,2 $\mu \mathrm{m}$. Estrias: 8-11/10 $\mu \mathrm{m}$.

O material registrado para o rio Descoberto foi identificado como Pinnularia gibba Ehrenberg, pois 
tanto as medidas, como a forma confere com a descrição de Krammer (1992), Lange-Bertalot \& Meltzeltin (1996), Krammer \& Lange-Bertalot (1986), Gasse (1986) bem como as ilustrações. Segundo Hustedt (1959) as medidas de comprimento encontram-se acima do observado neste trabalho, mas apresenta, em suas figuras, dois morfotipos de $P$. gibba e comenta que o tamanho da área axial não é uma característica que serve para diferenciar as variedades do táxon, mas que considera o formato da valva como um bom indicador. Ainda neste mesmo sentido, Krammer \& Lange-Bertalot (1986); Germain (1981) e Patrick \& Reimer (1966) comentam o alto polimorfismo e a circunscrição pouco definida para esta espécie.

Distribuição para o Estado de Goiás e DF. Goiás: Goiânia (Macedo-Saidah et al. 1987); Goiânia (Campos \& Macedo-Saidah1985); Itajá (Contin \& Oliveira 1993). DF: Planaltina (Souza \& Moreira Filho 1999b).

Material examinado: BRASIL. Distrito Federal: a jusante da confluência do rio Melchior com o rio Descoberto, 23/VI/2003, M.G.M. Souza (UB 1498); 7/VII/2003 (UB 1509).

Pinnularia microstauron (Ehrenberg) Cleve, Acta Soc. Fauna Fl. Fennica, 8(2):28, 1891.

Stauroptera microstauron Ehr. Phys. Abh. Akad.

Wiss. Berlin, p.423, pr. 1(4), fig. 1. 1843.

Fig. 15

Valva linear a lanceolada, margens levemente convexas na região mediana; extremidades rostradas, subcapitadas arredondadas; área axial estreita; área central rombóide, atingindo apenas uma das margens valvar; estrias encurtando-se na região central em um dos lados da valva; rafe filiforme; extremidades proximais da rafe voltadas para a mesma direção; extremidades distais da rafe em forma de foice; estrias transapicais alveoladas radiadas na região mediana e, em direção às extremidades, levemente paralelas a convergentes. Comprimento: 35,5-52 $\mu \mathrm{m}$. Largura: 6,6-9,1 $\mu \mathrm{m}$. Estrias: 9-11/10 $\mu \mathrm{m}$.

Em comparação com Jensen (1985) e Patrick \& Reimer (1966), tanto a descrição como a morfometria e a figura de Pinnularia microstauron (Ehrenberg) Cleve, excetuando o número de estrias que está ligeiramente acima do encontrado, confere com o observado no rio Descoberto. O táxon descrito para o rio Descoberto assemelha-se ao morfotipo $3 \mathrm{de}$ P. microstauron (Ehrenberg) Cleve var. microstauron descrito por Krammer (1992). Difere-se de Pinnularia submicrostauron Schoeter graças a área central nitidamente circular ao invés de rombóide e as estrias não são interrompidas na área central. Ainda, segundo Krammer (1992), P. microstauron se assemelha a Pinnularia brebissonii (Kützing) Rabenhorst, porém esta apresenta valva elíptica e extremidades cuneadas, o que não ocorre com o outro táxon. No mais, as estrias são interrompidas na região mediana. P. microstauron pode ser diferenciada de $P$. interrupta $\mathrm{Wm}$. Smith e Pinnularia gibba Ehr. através de microscopia eletrônica (Krammer 1992).

Distribuição para o Estado de Goiás e DF. Goiás: Goiânia (Macedo-Saidah et al. 1987); Itajá (Contin \& Oliveira 1993). DF: citação pioneira.

Material examinado: BRASIL. Distrito Federal: córrego Barrocão, 23/VI/2003, M.G.M. Souza (UB 1489); a jusante da barragem rio Descoberto, 7/VII/2003, M.G.M. Souza (UB 1507).

Pinnularia similis Hustedt, Hustedt 1934, in Schmidt et al., Atlas der Diatom. pr. 385, fig. 10-12, 18471959.

Fig. 16

Valva linear a elíptica, margens onduladas; extremidades subcapitadas cuneadas; área axial estreita; área central rombóide, atingindo as margens da valva; estrias encurtando-se na região central em ambos os lados da valva; rafe levemente lateral; extremidades proximais da rafe voltadas para a mesma direção; extremidades distais da rafe em forma de baioneta; estrias transapicais alveoladas radiais na região mediana e, convergentes nas extremidades. Comprimento: $52,8 \mu \mathrm{m}$. Largura: $8 \mu \mathrm{m}$. Estrias: $11 / 10 \mu \mathrm{m}$.

O material identificado concorda com a descrição de Krammer (2000) e assemelha-se com a figura 2 da prancha 172 de Metzeltin, Lange-Bertalot \& GarcíaRodríguez (2005), embora não apresentem a descrição para a espécie.

Distribuição para o Estado de Goiás e DF: citação pioneira para o Estado de Goiás e DF.

Material examinado: BRASIL. Goiás: a montante da confluência do rio Melchior com o rio Descoberto, 7/VII/2003, M.G.M. Souza (UB 1508).

Pinnularia subanglica Krammer, Diatoms of Europe. 1: p. 108. pr. 84, fig. 1-7, 2000.

Fig. 17

Valva linear; extremidades capitadas, área axial estreita; área central larga, rombóide com fascia; rafe filiforme; extremidades proximais da rafe voltadas 


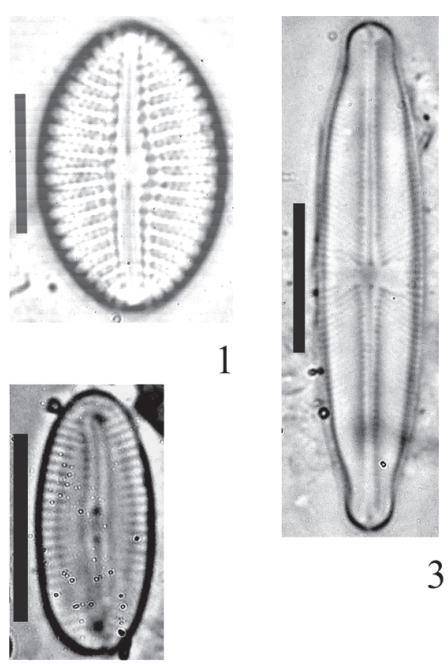

2

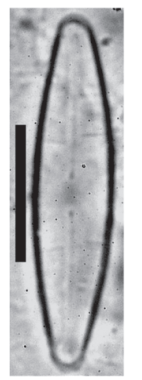

9

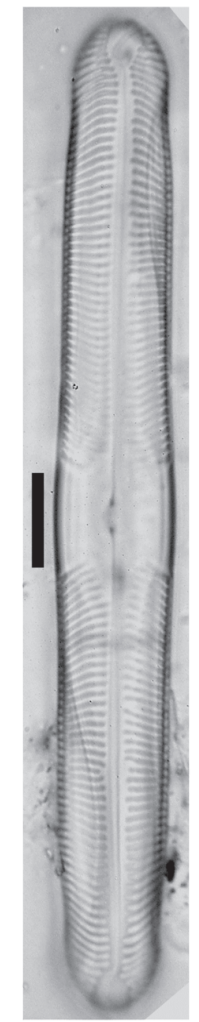

10

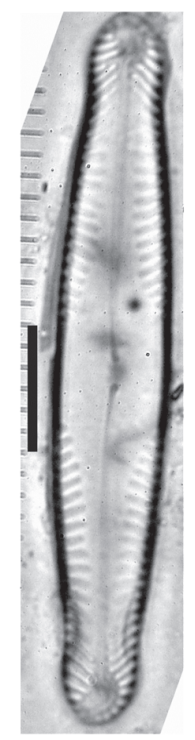

11

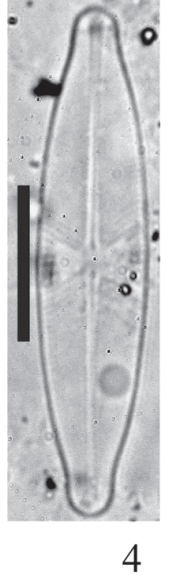

4

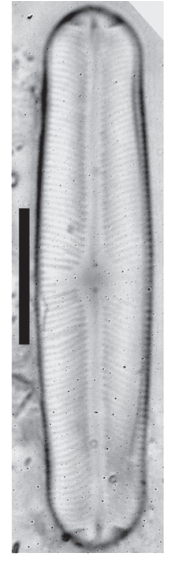

5
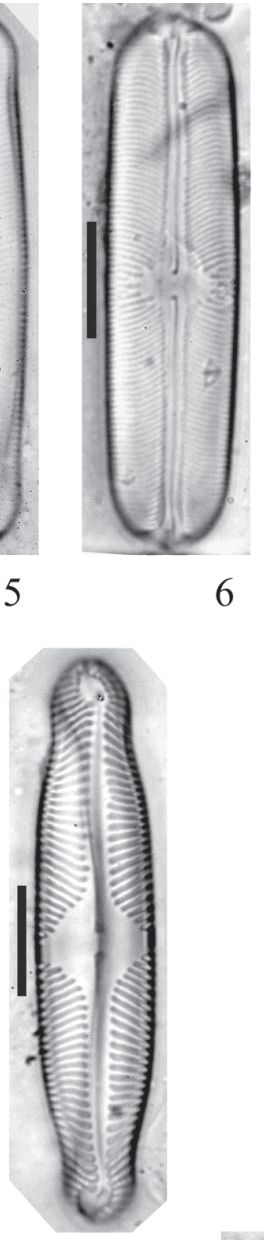

13

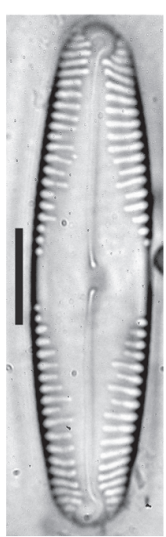

14

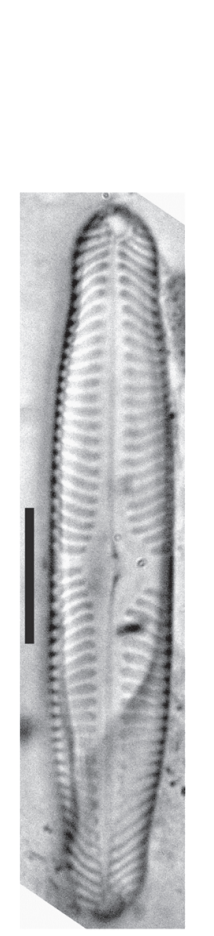

15
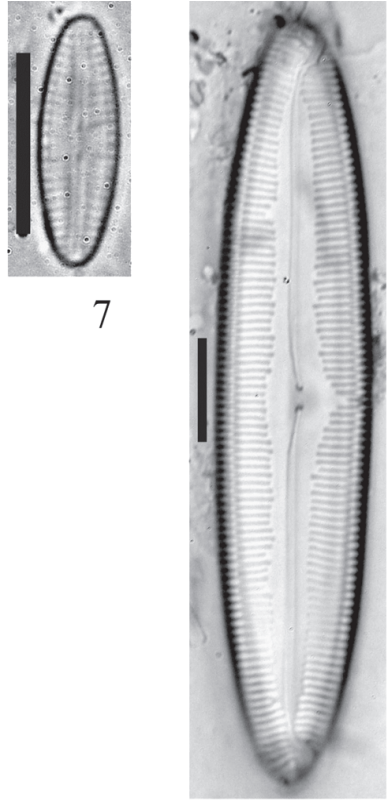

8

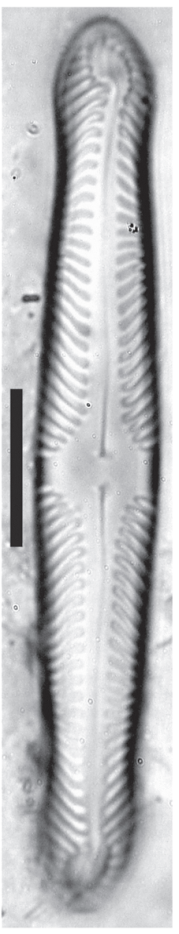

16

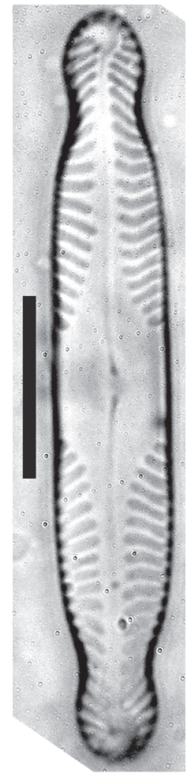

17

Figura 1. Diploneis subovalis Cleve. 2. Fallacia insociabilis (Krasske) D.G. Mann. 3-4. Sellaphora nyassensis (O. Muller) D.G. Mann. 5. Sellafora pupula (Kützing) Mereschkowsky. 6. Sellafora rectangularis (Gregory) Lange-Bertalot \& Metzeltin. 7. Sellafora seminulum (Grunow) D.G. Mann. 8. Caloneis butantana Krasske. 9. Caloneis hyalina Hustedt. 10. Pinnularia amazonica Metzeltin \& LangeBertalot. 11. Pinnularia brauniana (Grunow) Mills. 12. Pinnularia crucifera Cleve-Euler. 13. Pinnularia divergens var. media Krammer. 14. Pinnularia gibba Ehrenberg. 15. Pinnularia microstauron (Ehrenberg) Cleve. 16. Pinnularia similis Hustedt. 17. Pinnularia subanglica Krammer. Escalas $=10 \mu \mathrm{m}$. 
para a mesma direção; poros centrais distintos; estrias transapicais radiadas na área central e, em direção às extremidades, convergentes. Comprimento: 35,5-44 $\mu \mathrm{m}$. Largura: 6,4-8,8 $\mu \mathrm{m}$. Estrias: 10-12/10 $\mu \mathrm{m}$.

O táxon encontrado para o rio Descoberto foi identificado como Pinnularia subanglica Krammer, por concordar com a descrição e ilustrações de Krammer (2000).

Distribuição para o Estado de Goiás e DF: citação pioneira para o Estado de Goiás e DF.

Material examinado: BRASIL. Distrito Federal: a jusante da barragem rio Descoberto, 23/VI/2003, M.G.M. Souza (UB 1496); a jusante da confluência do rio Melchior com o rio Descoberto, 7/VII/2003, M.G.M. Souza (UB 1509); Goiás: a montante da confluência do rio Melchior com o rio Descoberto, 23/VI/2003, M.G.M. Souza (UB 1497); 7/VII/2003 (UB 1508).

\section{Agradecimentos}

À equipe de campo do CTHidro do Programa de Pós Graduação em Tecnologia Ambiental e Recursos Hídricos da Faculdade de Tecnologia, Departamento de Engenharia Civil e Ambiental (UnB) sob coordenação do Prof. Dr. Sérgio Koide; ao Núcleo de Estudos Limnológicos da Universidade de Brasília (NEL/UnB); ao Programa de PósGraduação em Botânica; ao Departamento de Botânica; ao Herbário da Universidade de Brasília; à CAPES pela Bolsa de Mestrado concedida à primeira autora.

\section{Referência bibliográficas}

Brassac, N.M. \& Ludwig, T.A.V. 2003. Fragilariaceae (Bacillariophyceae) de rios da bacia do Iguaçu Estado do Paraná, Brasil. Revista Brasileira de Botânica 26(3): 311-318.

Brassac, N.M. \& Ludwig, T.A.V. 2005. Amphipleuraceae and Diploneidaceae (Bacillariophyceae) from Iguaçu basin river, PR, Brazil. Acta Botanica Brasilica 19(2): 359-368.

Campos, I.F.P. \& Macedo-Saidah, F.F. 1985. Flórula da Represa da Escola de Agronomia da Universidade Federal de Goiás, Goiânia, Goiás, Brasil. Pp. 839-857. In: Anais do XXXVI Congresso Nacional de Botânica. v.2, Brasília, IBAMA/SBB.

Campos, I.F.P.; Rizzo, J.A. \& Pereira, H.D. 1990. Algas do Estado de Goiás. Pp. 9-18. In: I.F.P. Campos (ed.). Flora do estado de Goiás, criptógamos. v.1, n. 1, Goiânia, Editora da UFG-EDUFG.
CODEPLAN - Companhia de Desenvolvimento do Planalto. 1984. Atlas do Distrito Federal. vol II. Brasília, DF.

Contin, L.F. \& Oliveira, R.J.M. 1993. Diatomáceas (Chrysophyta - Bacillariophyceae) em águas termais: Lagoa Santa - Município de Itajá - Goiás. Pp. 7-35. In: I.F.P. Campos (ed.). Flora dos Estados de Goiás e Tocantins, criptógamos. v.2, n. 1, Goiânia, Editora da UFG-EDUFG.

Gasse, F. 1986. East African Diatoms - taxonomy, ecological distribution. Bibliotheca Diatomologica 11: 1-201.

Germain, H. 1981. Flore dês diatomées. Paris, Boubée.

Hustedt, F. 1959. Die kieselalgen. In: L. Rabenhorst (19611966) Kryptogamen - flora. Lipzig, Akademischen Verlagsgesellschaft, v.7, parte 3.

Jensen, N.C. 1985. Hustedt's “Die Kieselalgen, 2. Teil”: the Pennate Diatoms. Koenigstein, Koeltz Scientific Books.

Krammer, K. \& Lange-Bertalot, H. 1986. Bacillariophyceae: Naviculaceae. In: H. Ettl; I. Gerloff; H. Heynig \& D. Mollenhauer (eds.). Süsswasserflora Von Mitteleuropa. Stuttgart, G. Fischer. v.2, n. 1.

Krammer, K. 1992. Pinnularia eine Monographie der europäischen taxa. Bibliotheca Diatomologica 26: 1-353.

Krammer, K. 2000. The genus Pinnularia. Diatoms of Europe 1: $1-703$.

Lange-Bertalot, H. \& Metzeltin, D. 1996. Indicators of Oligotrophy - 800 taxa representative of three ecologically distinct lake types. Iconographia Diatomologica 2: 1-390.

Lange-Bertalot, H.; Külbs, K.; Lauser, T.; Nörpel-Schempp, M. \& Willmann, M. 1996. Dokumentation und Revision der von Georg Krasske beschriebenen Diatomeen-Taxa. Iconographia Diatomologica 3: 1-358.

Macedo-Saidah, F.E.; Nascimento, M.R.R. \& Campos, I.F.P. 1987. O plâncton das águas do Rio Meia-Ponte no município de Goiânia, Goiás, Brasil. Nerítica 2: 105-118.

Metzeltin, D. \& Lange-Bertalot, H. 1998. Tropical Diatoms of South America I. Iconographia diatomologica 5: 1-695.

Metzeltin, D.; Lange-Bertalot, H. \& García-Rodríguez, F. 2005. Diatoms of Uruguay. Iconographia diatomológica 15: 1-736.

Moreira Filho, H. \& Valente-Moreira, I. 1981. Avaliação taxonômica e ecológica das diatomáceas (Bacillariophyceae) epifíticas em algas pluricelulares obtidas nos litorais do Paraná, Santa Catarina e São Paulo. Boletim do Museu Botânico Municipal 47: 1-17.

Patrick, R. \& Reimer, C.W. 1966. The diatoms of the United States. Monographs of the Academy of Natural Sciences of Philadelphia 1: 1-688.

Prygiel, J. \& Coste, M. 1999. Guide méthodologique pour la mise en ceuvre de I'Indice Biologique Diatomées. Agence de l'Eau Artois-Picardie CEMAGREF.

Round, F.E.; Crawford, R.M. \& Mann, D.G. 1990. The diatoms - Biology and morphology of the genera. Cambridge, Cambridge University Press. 
Simonsen, R. 1974. The diatom plankton of the Indian Ocean expedition of R/V "Meteor", 1964-1965. Meteor. Forschungsergebnisse Reihe D-Biologie 19: 1-66.

Souza, M.G.M. \& Compère, P. 1999. New diatom species from the Federal District of Brazil. Diatom Research 14(2): 357-366.

Souza, M.G.M. \& Moreira Filho, H. 1999a. Diatoms (Bacillariophyceae) of two aquatic macrophyte bancks from Lagoa Bonita, Distrito Federal, Brazil, I: Thalassiosiraceae and Eunotiaceae. Bülletin du Jardin Botanique National de Belgique 67: 259-278.
Souza, M.G.M. \& Moreira Filho, H. 1999b. Diatoms (Bacillariophyceae) of two aquatic macrophyte bancks from Lagoa Bonita, Distrito Federal, Brazil, II: Navicula sensu lato and Pinnularia. Bülletin du Jardin Botanique National de Belgique 67: 279-288.

Stoermer, E.F. \& Smol, J.P. 1999. The Diatoms: applications for the environmental and earth sciences. Cambridge, Cambridge University Press.

Van den Hoek, C.; Mann, D.G. \& Jahns, H.M. 1995. Algae: an introduction to phycology. Cambridge, Cambridge University Press. 